FORMATION Formation emploi

Revue française de sciences sociales

129 | Janvier-Mars 2015

Pêle-Mêle

\title{
Les enjeux d'une évaluation commanditée : Immersion au sein d'une recherche-intervention en sciences de l'éducation
}

Issues of an ordered evaluation - Immersion within a research-intervention in education sciences

Die Herausforderungen von Auftrags-Evaluierungen : Immersion in eine erziehungswissenschaftlichen Interventionsforschung

Los desafíos de una evaluación por encargo : Inmersión en una investigaciónintervención en ciencias de la educación

\section{Lucie Aussel}

\section{OpenEdition}

Journals

Édition électronique

URL : http://journals.openedition.org/formationemploi/4374

DOI : 10.4000/formationemploi.4374

ISSN : 2107-0946

Éditeur

La Documentation française

Édition imprimée

Date de publication : 1 mars 2015

Pagination : 87-105

ISSN : 0759-6340

Référence électronique

Lucie Aussel, «Les enjeux d'une évaluation commanditée : Immersion au sein d'une rechercheintervention en sciences de l'éducation », Formation emploi [En ligne], 129 | Janvier-Mars 2015, mis en ligne le 01 mars 2017, consulté le 30 octobre 2020. URL : http://journals.openedition.org/ formationemploi/4374 ; DOI : https://doi.org/10.4000/formationemploi.4374 


\title{
Les enjeux d'une évaluation commanditée
}

\section{Immersion au sein d'une recherche-intervention en sciences de l'éducation}

\begin{abstract}
LUCIE AUSSEL
Post-doctorante au LLA - Creatis (Lettres, Langues, Arts - Création, recherche, émergence en arts, textes, images, spectacle), université de Toulouse. Chercheure associée UMR EFTS (Education, Formation, Travail, Savoirs), université de Toulouse

Résumé

Les enjeux d'une évaluation commanditée. Immersion au sein d'une rechercheintervention en sciences de l'éducation

Cet article présente une réflexion épistémologique et méthodologique sur l'évaluation commanditée. Les résultats d'une recherche-intervention menée, de 2009 à 2013, dans le cadre d'un partenariat entre une instance politique et des chercheurs en sciences de l'éducation viennent étayer les propos. Les recherches commanditées, notamment lorsqu'elles sont financées par des décideurs politiques, nécessitent-elles une prise en charge particulière ? À travers l'exemple de cette recherche, quatre enjeux interdépendants seront mis au jour : éthique, stratégique, méthodologique et praxéologique.
\end{abstract}

Mots clés :

Expérimentation sociale, science de l'éducation, évaluation, chercheur, méthodologie

Abstract

Issues of an ordered evaluation. Immersion within a research-intervention in education sciences

This paper presents an epistemological and methodological reflection on the ordered evaluation. The results of a research conducted from 2009 to 2013 as part of a partnership between a political authority and researchers in education sciences come substantiate the remarks. Do ordered researchs, especially when financed by policy makers, need a special treatment? Using the example of this research, four issues will be revealed: ethics, strategic, methodological and praxeological.

\section{Keywords:}

Social experiment, educational science, evaluation, researcher, methodology Journal of Economic Litterature: C 9, Y 80

Traduction : Auteure. 
Nous proposons ici une réflexion épistémologique et méthodologique sur l'évaluation, lorsque des chercheurs choisissent de répondre à une demande d'évaluation qui sera négociée puis contractualisée. Nos propos s'appuient sur les résultats d'une recherche-intervention, menée de 2009 à 2013, dans le cadre d'un partenariat entre une instance politique et des chercheurs en sciences de l'éducation.

Le Haut-Commissariat à la Jeunesse, aux Sports et à la Vie associative, devenu depuis Fonds d'expérimentations pour la jeunesse (FEJ), a lancé un appel à projets destiné à soutenir et promouvoir des expérimentations sociales. Elles visaient la résolution de problèmes (décrochage et orientation) concernant une partie de la jeunesse française. Cet appel fut adressé à la fois aux praticiens (élaboration d'un dispositif innovant) et aux équipes de recherche (évaluation dudit dispositif) ; les deux composantes, expérimentation et évaluation, ayant formulé une réponse commune à l'appel d'offres.

Les recherches commanditées comme celle-ci, notamment lorsqu'elles sont financées par des décideurs politiques, nécessitent-elles une prise en charge particulière ? Si on peut répondre par l'affirmative à cette question, elle mérite néanmoins d'être posée. De quelle prise en charge parlons-nous ? Quelle est la nécessité de faire de la recherche autrement lorsque celle-ci est collaborative ${ }^{1}$ et a fortiori commanditée?

À travers l'exemple que nous venons d'évoquer, nous soumettons quelques pistes de réflexion. Ainsi, après avoir évoqué l'état de la recherche sur l'évaluation de dispositif de formation, nous présenterons le cas sur lequel s'appuie notre argumentation. Nous mettrons en perspective les enjeux d'une telle démarche à travers l'exemple de la recherche-intervention que nous avons choisi pour répondre à notre interrogation. Nous développerons ensuite quatre parties relatives à ces différents enjeux : éthique (posture du chercheur); stratégique (négociation de l'objet de connaissance), méthodologique, et enfin praxéologique (accompagnement de la décision politique par la restitution des résultats).

\section{Des recherches sur l'évaluation de dispositifs de formation}

Leclercq (2008, p. 74) donne une définition des dispositifs de formation : " un projet pour autrui, un moyen d'atteindre un objectif ou de tendre vers un but de manière intentionnelle". Il ajoute : " toute aussi prégnante est l'idée que le projet pour autrui ne s'actualise que sil est reconfiguré, redéfini, réapproprié. Pour le dire autrement, la conception d'un dispositif se poursuit effectivement dans l'usage. " L'évaluation de dispositif de formation est un champ de recherche encore en évolution. Les publications francophones présentent comme objet d'évaluation des "programmes " (Figari \& Tourmen, 2006), "des systèmes éducatifs "

1. Ce terme est employé dans le sens de Desgagné (1997, p. 372) lorsqu’il soutient que « la recherche collaborative suppose la co-construction d'un objet de connaissance entre un chercheur et des praticiens". 
(Lafontaine \& Simon, 2008) des "politiques publiques ", parfois mêmes des "dispositifs" (Bedin \& Talbot, 2010 ; Broussal, 2010 ; Figari, 2008) employant l'un ou l'autre des termes.

Il existe une plus forte uniformité dans la littérature anglophone, le terme "program " (Wong-Ratcliff, Powell Ezell, Cage, \& Chen, 2011) est très largement employé, notamment aux Etat-Unis, où " l'évaluation de programme et de projet y connaît un développement important" (Dussault, 2008, p. 61). Le terme "device" est plus rare en éducation pour désigner le dispositif ; il correspond davantage à des dispositifs techniques (Mohd Suki \& Mohd Suki, 2011). Peraya et Peltier (2012, p. 112) affirment que la notion de dispositif de formation s'est « imposée progressivement à partir des années 1970 " pour se généraliser, selon Albero (2010), dans les années 1990. Peraya et Peltier (op. cit., p. 112), mentionnent que son succès serait dû à "l'influence de la technologie de l'instruction, de la "technologisation" de l'apprentissage, mais aussi par la pression constante de l'industrialisation de la formation". Albero (op. cit., p. 49) suggère que ce développement est lié à l'adoption d'une "perspective plus écologique " de la formation, dans laquelle "il y a une ouverture à l'activité effective des sujets et au sens qu'ils lui accordent, aux espaces de travail dans lesquels ils opèrent, à la diversité des projets et objectifs de formation ".

L'émergence des recherches sur l'évaluation de dispositifs de formation dans ce partenariat politique-recherche va être abordée dans cette partie. Ainsi, un premier point rappelle le contexte des évaluations de politiques publiques dans lesquelles elles s'inscrivent, puis un second détaille leurs particularités.

\subsection{D'un contexte d'évaluations de politiques publiques...}

En France, c'est à la fin des années 1960, avec l'accroissement des dépenses publiques, que se développe le souci de l'efficacité de l'action publique. L'évaluation s'est étendue en tant qu'évaluation expost, en relation avec les politiques de modernisation de l'État. Le dispositif institutionnel d'évaluation dans l'administration s'étoffe au début des années 1990, pour faire de l'évaluation un élément central de la prise de décision gouvernementale et du débat démocratique (Perret, 2001). Dans les années 1990, deux décrets importants relatifs à l'évaluation des politiques publiques sont ratifiés. Celui du 22 janvier 1990 permet la création d'un Conseil scientifique de l'évaluation ; celui du 18 novembre 1998, celle d'un Conseil national de l'évaluation. Ces deux instances encadrent ces évaluations; elles déterminent les politiques à évaluer, ainsi que les évaluateurs en charge de la mission. En 1992, un "Petit guide de l'évaluation" est créé par le Conseil scientifique de l'évaluation. Il s'agit d'un support méthodologique pour la pratique évaluative.

Dès le début, les chercheurs en sciences sociales occupent des postes d'évaluateurs. Le "Petit guide " précise que la pratique de l'évaluation ne peut s'apparenter à de la recherche pure, car même si les équipes de chercheurs sont autonomes, elles répondent à une commande. Ils font ainsi de la recherche évaluative un genre hybride, entre la recherche et une pratique 
professionnelle d'évaluation (mise en œuvre par des consultants, experts). C'est dans ce contexte de recherche commanditée qu'émerge l'évaluation de dispositifs.

\section{2 ... aux recherches sur l'évaluation de dispositifs de formation}

Les pratiques évaluatives liées aux dispositifs éducatifs représentent un nouveau mode de fonctionnement de la recherche française. Elles induisent une gestion par projet et peuvent devenir des missions dont la temporalité échappe aux chercheurs, leur permettant l'obtention d'un budget et d'un cadre contractualisé avec des financeurs décideurs ${ }^{2}$.

Les évaluations de dispositifs sont menées dans les courants de recherches orientées vers l'amélioration des pratiques et l'élaboration de formation. Lorsqu'elles sont commanditées, elles sont médiatisées par la commande d'un acteur décideur (politique/administratif, etc.) qui souvent, par le biais d'appels à projets, passe un contrat avec des chercheurs chargés de l'évaluation. Cette commande permet à la fois à l'évaluation d'exister en créant son cadre de réalisation, mais aussi détermine les objectifs et donc les missions du chercheur-évaluateur. C'est un moment clé, "une pratique à vocation collaborative" (Bedin \& Talbot, 2010) qui appelle à la négociation les différentes parties du projet. Il s'agit pour les commanditaires de transmettre les enjeux de l'évaluation et pour les futurs évaluateurs de bien cerner à la fois l'ensemble des attendus et de lever les "points aveugles» (ibid.).

Il existe deux orientations de recherche en la matière :

La première est centrée sur les produits du dispositif ; elle est perçue comme une expertise par les commanditaires. Cela signifie qu'elle s'apparente aux pratiques anglo-saxonnes dites d'accuntability que Lafontaine et Simon (2008, p. 103) qualifient d' "exigence de rendre des comptes pour les politiques publiques", pour lesquelles des "sommes considérables vont être allouées aux recherches visant à évaluer l'efficacité des systèmes d'enseignement " (De Ketele, 1993, p. 73). L'efficacité est obtenue par comparaison des objectifs fixés au démarrage de l'évaluation et de ses effets propres (Perret, op. cit.). Cette façon de procéder est très répandue dans le monde anglo-saxon où se développe l'éducation basée sur la preuve (EBP) qui prend appui sur des recherches estimées fiables selon les standards de la recherche quantitative. Saussez et Lessard (2009) présentent deux méthodes phares de l'EBP ; la première permet d'établir la preuve de ce qui fonctionne avec un système de production de la preuve (l'essai contrôlé randomisés ), la seconde permet de la rendre disponible avec "un système d'analyse,

2. Il s'agit, par exemple, d'acteurs à l'origine de la demande d'évaluation et qui en sont les principaux destinataires. Il leur incombe ensuite de prendre les décisions politiques en lien ou pas avec les recommandations de l'évaluation.

3. Cette méthode suppose la composition d'un groupe expérimental (bénéficiant d'une expérimentation), d'un groupe contrôle (n'en bénéficiant pas) selon un tirage au sort aléatoire, et la mise en oeuvre d'observations pré-expérimentales et post-expérimentales. 
d'évaluation, de synthèse et de diffusion de la preuve (la revue systématique de recherche) " " (ibid., p. 112).

La seconde orientation est dite écologique ; elle est défendue notamment par Younès (2010) qui établit l'insuffisance d'une approche uniquement quantitative de l'évaluation. Pour elle, l' "approche "quantitativiste" tend à évacuer le fait que les dispositifs sont aussi des univers symboliques participant d'une construction personnelle et collective" (ibid., p. 1). Elle préconise une approche plus globale tenant compte de la complexité des dispositifs et de leurs différents composants, en lien avec les « conditions environnementales " de leur réalisation. L'auteure propose de s'intéresser à l'analyse des processus du dispositif et insiste sur la nécessité "d'articuler différents niveaux d'explicitation: intraindividuel, interindividuel, positionnel et idéologique" (ibid., p. 2). Ainsi, "l'évaluation de dispositif éducatif correspondrait à l'évaluation d'un système situé d'interactions entre les constituants de ce dispositif" (ibid., p. 2). Broussal (2010, p. 9) témoigne également de l'importance de l'approche multidimensionnelle et pointe les limites des évaluations centrées sur les produits "laissant dans l'ombre les débats publics qui concernent les valeurs".

\section{De la présentation du cas au questionnement de recherche}

La problématique relative à la recherche en situation de commande va être développée à partir de son contexte. Ainsi, le cahier des charges et le dispositif de formation au cour de l'évaluation seront décrits. Ensuite, les enjeux de ce type d'évaluation seront mis en avant à travers la démarche de recherche-intervention choisie dans ce cas pour répondre à la commande.

\subsection{Evaluer un dispositif innovant de formation dans l'enseignement supérieur agricole}

En 2009 est lancé l'appel à projets qui concerne cette recherche, "Génération active ". Il fait partie de la "première vague " composée de quatre axes. Ceux-ci promeuvent des expérimentations sociales accompagnées d'évaluations externes, c'est-à-dire réalisées par des équipes ne faisant pas partie du montage des expérimentations. Le projet auquel nous nous sommes associés s'inscrit dans l'axe 1 « Réduire les sorties prématurées du système de formation initiale». Il fait partie du quatrième des sept $\mathrm{t}^{5}$ programmes de cet axe : "Approfondir le

\footnotetext{
4. Selon Saussez et Lessard (op. cit., p. 113) il s'agit d'une méthode de gestion de l'information évaluative structurée en quatre étapes : formuler une question et spécifier les critères de recherche ; réaliser une recherche systématique ; évaluer la qualité des preuves ; formuler une réponse claire à la question posée.

5. 1. : sécuriser l'orientation vers l'alternance et prévenir les ruptures 2 . : prévenir le décrochage scolaire et organiser les collaborations pour suivre les jeunes décrocheurs 3. : développer les incitations au maintien
} 
dispositif d'orientation active ". Les praticiens-expérimentateurs devaient répondre à deux impératifs : proposer un dispositif permettant une " évaluation rigoureuse ", c'est-à-dire une évaluation se reconnaissant dans le paradigme de la mesure ; s'inscrire dans un territoire de niveau méso ou macro.

Pour les évaluateurs, une orientation méthodologique était également préconisée $c f$. infra. Elle devait mettre en évidence les effets des dispositifs afin d'envisager ou non leur généralisation.

Le dispositif expérimental évalué était destiné à des étudiants préparant un Brevet de technicien supérieur agricole (BTSA). L'objectif de l'expérimentation était de «permettre aux apprenants d'approcher les métiers autrement, de réfléchir puis d'agir pour réussir leur orientation puis leur insertion professionnelle ${ }^{6} »$. Portée par une association spécialiste du recrutement et de l'insertion des cadres et techniciens du secteur agricole, ce dispositif s'est déroulé sur les deux années de formation des étudiants. Au cours de leur première année, (2009/2010), les étudiants ont suivi un module devant leur permettre de découvrir les fonctions de l'entreprise et de décrypter les profils métiers de leur secteur professionnel. Durant leur deuxième année (2010/2011), ils ont participé au second module, axé sur les démarches de recherche d'emploi. L'expérimentation a été mise en place sur l'ensemble du territoire national français, auprès de soixante-huit classes (770 étudiants) préparant quatre spécialités de l'enseignement supérieur agricole 7 .

Ainsi, l'objet de notre évaluation est tout d'abord un dispositif. Il est la création, par des individus (une association), d'un support de formation venant concrétiser l'intention d'améliorer l'insertion et l'orientation professionnelle de ces étudiants. Il est en tension entre la volonté d'émancipation (des étudiants) et d'efficacité (du dispositif). Ainsi que le mentionne Audran (2007, p. 11) « la particularité des dispositifs est de renvoyer à un espace de liberté et de contrainte, mi-écrit, mi-vécu».

Il constitue ensuite un dispositif innovant. En effet, ce projet qui a commencé par suivre le cycle ordinaire des créations de formations au sein de l'association ${ }^{8}$, s'est d'abord développé pour un public d'ingénieurs. Ici, le cycle classique des innovations au sein de l'organisation est rompu par le statut expérimental que prend le dispositif lorsqu'il s'agit de le transposer aux étudiants de BTSA. S'adresser à ce public plus jeune, dès la première année de leur formation, a nécessité la création de nouveaux outils et l'adaptation de la trame de la for-

dans le système scolaire 5. : organisation intégrée de coordination des acteurs de l'orientation scolaire et professionnell 6. : améliorer les dispositifs d'orientation et lutter contre l'échec scolaire 7. : accompagnement et insertion professionnelle des étudiants qui décrochent.

6. Annexe 1 p. 8 de la Convention pluriannuelle de l'expérimentation.

7. Les quatre options parmi les dix-sept existantes, soit Analyse et Conduite des Systèmes d'Exploitation (ACSE), Gestion et Protection de la Nature (GPN), Service en Espace Rural (SER) et Sciences et Technologies des Aliments (STA), ont participé à l'expérimentation

8. Qui a une organisation stabilisée pour faire émerger des innovations. 
mation à leur orientation et leurs débouchés. Enfin, ce dispositif innovant est un dispositif de formation; il correspond au "dispositif institué» de type 1 défini par Audran (2010). Sa dimension macro-environnementale et son origine politique visant à résoudre une situation problème et donc à véhiculer la norme sociétale de l'emploi, tendent à le rapprocher de cette définition.

\subsection{Concilier posture du chercheur et réponse à une commande ?}

La démarche de recherche-intervention a été choisie pour répondre à la commande précédemment développée. Elle est basée sur un principe d'articulation et de réciprocité entre ses deux composantes : la recherche (ici sur l'évaluation de dispositif de formation) et l'intervention (pratique évaluative du dispositif innovant de formation) qui s'alimentent mutuellement.

L'évaluation étudiée s'inscrit dans une demande sociale entendue comme un "système d'attentes de la société [demande ici relayée par une instance politique] à l'égard de problèmes quotidiens [ici l'orientation et l'insertion des jeunes]"(Castel, 2004, p. 70). Elle est négociée et s'adresse à des chercheurs, devenant, lorsqu'ils y répondent, des chercheurs-intervenants.

Cette recherche commanditée interroge donc sur la place de la recherche au sein de ce type de demande, dont les attendus répondent à un cahier des charges précis tributaire de la subvention. Comment assurer une posture de chercheur tout en répondant à la commande ?

Ensuite, dans ce cas d'une commande portant sur l'évaluation d'un dispositif innovant de formation, préconisant une méthodologie quantitative avec randomisation et comparaison d'un groupe expérimental et d'un groupe contrôle, la marge d'autonomie des chercheurs dans la conduite de leur recherche est questionnée. Répondre à un appel à projets comme celui-ci permet-il de développer son champ de spécialité et de participer à la construction des connaissances?

La première interrogation renvoie à des enjeux éthiques et stratégiques. En effet, choisir d'initier une recherche en réponse à ces demandes signifie s'engager dans la cité et ainsi accepter de contribuer à la construction de connaissances " pour» et "sur» l'action (Marcel, 2010), en vue de répondre à un besoin. En effet, la participation des chercheurs au discours public correspond à un enjeu éthique, puisqu'il s'agit de se positionner par rapport à l'action que l'on mène (Ethique de la responsabilité, Ricœur, 1996).

De plus, dans le prolongement de ce premier enjeu, le second est nécessairement stratégique car, dans ce cas, s'engager signifie travailler avec et pour les acteurs. Il s'agit de pouvoir affirmer les fondements de la recherche et de ne pas négliger leur importance.

La seconde interrogation renvoie, quant à elle, à des enjeux méthodologiques et praxéologiques. Comme mentionné supra, le cahier des charges contraint l'évaluation. Ainsi le premier enjeu, méthodologique, nécessairement lié à l'enjeu stratégique, interroge la marge de manœuvre de l'équipe de recherche vis-à-vis du cadre qui lui est imposé (la randomisation 
par exemple) et de ses intérêts scientifiques. Ensuite, la question des résultats produits et adressés à des décideurs mais aussi aux partenaires (ici praticiens-expérimentateurs) nous semble être primordiale. Une réflexion d'ordre praxéologique sur le statut de ces résultats sera donc engagée.

\section{De l'engagement du chercheur 3 à la négociation de la commande}

Les deux premiers enjeux énoncés dans la problématisation vont être développés ici : l'enjeu éthique concerne la posture du chercheur-intervenant ; l'enjeu stratégique aborde le passage de la demande à la commande à travers la question de la négociation.

\subsection{La posture du chercheur-intervenant}

La posture renvoie, selon Ardoino (1990, p. 28), à " un système d'attitudes et de regards vis-à-vis des partenaires, des situations, des objets, dans le cadre de recherches ou de pratiques sociales". Pour étayer davantage la construction de cette posture, nous nous référons à Marcel et Nunez Moscoso (2012) qui interrogent la posture des chercheurs en éducation et proposent une nouvelle figure, celle du "chercheur-citoyen ". Pour ces auteurs, elle repose sur le nécessaire engagement du chercheur dans la cité, celui-ci n'étant synonyme ni de militantisme ni d'expertise à assener au nom de la science, mais signifiant plutôt de prendre part raisonnablement aux demandes sociales.

Les auteurs mentionnent un point qui nous paraît essentiel : l'utilité sociale des chercheurs. Il n'est pas question de contribuer à une théorisation utilitariste de la recherche, mais de plaider en faveur d'un engagement citoyen dans ce type de démarche. Pour cela, le chercheur ne se muera ni en expert ${ }^{9}$ ni en consultant. Il n'a pas vocation à délivrer des "solutions " tirées d'études précédentes, créant ainsi le problème évoqué par Bru (1998) de "l'extension des résultats scientifiques hors de leur cadre de théorique ou expérimental d'origine et leur utilisation comme preuve pour valider une pratique " (p. 48). De même, le chercheur n'a pas non plus pour mission de produire des recettes pour l'action (prescriptions).

La posture du chercheur-intervenant dans le cas de notre recherche a été celle de chercheurs-évaluateurs. La posture sera analysée selon la temporalité suivante : avant le

9. Nous distinguons le chercheur de l'expert et nous plaidons pour une posture de chercheur-intervenant. Pour Pesche (2012, p. 9) qui mène une réflexion sur les recherches réalisées à partir de réponse à des demandes, l'expertise peut être conçue comme la sélection de connaissances en vue d'aider à la prise de décision. Ainsi, l'expertise doit permettre de "fournir rapidement une ou des réponses sur le stock de connaissances disponibles»(ibid.). 
démarrage du projet (les prémices d'un engagement) et pendant la mise en place du dispositif (mise en œuvre d'une distanciation construite) ${ }^{10}$.

En amont du projet, la posture se dessine avec le choix d'un engagement. En effet, s'investir dans cet appel à projets signifiait, d'une part, mener une thèse sur une thématique en lien avec nos préoccupations scientifiques et, d'autre part, éprouver un type de recherche que nous ne connaissions pas et qui posait à nos yeux la question de l'utilité sociale du chercheur. Nous souhaitions contribuer à la production de connaissances sur les pratiques des acteurs du système éducatif et sur la place du chercheur dans la société. De plus, participer à cette recherche nous permettait de travailler dans des conditions que nous jugions "bonnes » (une thèse financée, rare en sciences de l'éducation hors $\mathrm{CDU}^{11}$ et bourses Cifre ${ }^{12}$ ). Durant le projet, nous avons pris soin d'expliciter à l'ensemble des acteurs de l'expérimentation les objectifs et finalités de la recherche-intervention. De plus, nous avons veillé à ne pas interférer dans la mise en place de l'expérimentation, afin de ne pas formuler de recommandations avant la fin de l'évaluation. Ainsi, malgré de nombreuses sollicitations, nous n'avons pas donné notre avis sur tel ou tel outil ni proposé des pistes d'amélioration au moment où nous étions sur le terrain, dans la phase de recueil des données et de compréhension du dispositif. La posture, cette distanciation construite ou le fait d' " être avec » sans interférer, a déçu certains de nos partenaires qui auraient souhaité un accompagnement progressif du dispositif de formation.

\subsection{De la demande à la commande}

Bedin (1999) considère " une structure invariante " (ibid., p. 94) des demandes d'étude : une double face, une double nature qu'il est important de connaître : une dimension émergente et une dimension latente. Ainsi, pour elle, "analyser la demande d'étude [...] tend à faire émerger les éléments contradictoires des demandes sociales, dont on suppose qu'ils structurent la demande formulée " (ibid., p. 97).

Cet appel à projets du FEJ, destiné aussi bien à la communauté des praticiens (de la jeunesse) qu’à la communauté scientifique (des sciences humaines et sociales), reflète, selon nous, une double dimension : sociale (émergente) et politique (latente).

En effet, la demande, par sa volonté de résoudre des situations problèmes, se place dans un axe social d'amélioration de l'existant. Elle poursuit un objectif politique particulier de gestion de la chose publique.

La forme de l'appel, avec la constitution de binômes praticiens/chercheurs, incités, pour les premiers, à produire une action efficace et, pour les seconds, à prouver cette efficacité,

10. Nous aborderons la question de la posture après la fin du projet à travers l'analyse d'un autre enjeu (praxéologique), plus loin.

11. Contrats Doctoraux Uniques.

12. Conventions Industrielles de Formation par la Recherche. 
tend à nous faire prendre conscience d'une volonté politique sous-jacente. Ce qui est mis en avant dans cet appel, c'est l'aspect social de la démarche : mettre en ouvre des fonds (humains et financiers) et mobiliser une partie des professionnels concernés par ces questions autour de la jeunesse. L'organisation de la gestion des fonds dans le discours politique vient ensuite ; pourtant, elle justifie un certain type de gouvernance et le recours à la légitimité des chercheurs pour prendre des décisions adressées au collectif. La dualité de la demande est exposée dans le Tableau 1 : émergente et latente, selon les catégories de Bedin (ibid.).

Tableau 1 : Dualité de la demande (émergente et latente) de l'appel à projets

\begin{tabular}{|c|c|}
\hline Demande émergente : sociale & Demande latente : politique \\
\hline $\begin{array}{c}\text { Résoudre des situations problèmes } \\
\text { Découvrir des dispositifs innovants } \\
\text { Éprouver leur efficacité } \\
\text { Décider (ou non) de leur généralisation }\end{array}$ & $\begin{array}{c}\text { Faire la preuve d'une gestion raisonnée de l'argent public } \\
\text { Légitimer la décision par un choix rationnel s'appuyant sur un proto- } \\
\text { cole scientifique }\end{array}$ \\
\hline
\end{tabular}

Source : Auteure.

La demande ainsi analysée peut être négociée afin d'être contractualisée et de devenir commande. Avant d'accéder à la contractualisation, l'étape cruciale de la négociation permet aux trois parties, commanditaires (FEJ), partenaires (praticiens-expérimentateurs), et chercheurs-intervenants, d'interagir et de poser les conditions de leur engagement dans le projet. Ainsi, nous avons mis en avant notre volonté de développer une recherche-intervention ; nous avons négocié l'intégration d'une doctorante à l'équipe afin de mettre en place l'évaluation et de mener une recherche sur l'évaluation des dispositifs de formation. Malgré la présence d'une « forte » règle du jeu, c'est-à-dire un cahier des charges prévoyant un type de méthode en particulier, la phase de négociation a permis à l'équipe de recherche de proposer des éléments de travail non envisagés, et de maintenir son cap scientifique. Cette étape s'est concrétisée ensuite par une phase de contractualisation, avec la construction de la commande. Selon Marcel (2012), la commande est un point de passage "entre deux phases de la recherche-intervention ", une passerelle. Deux phases la caractérisent : la "formalisation" et "l'adaptation" (ibid.). La première renvoie au "processus de transformation de la demande en commande formalisée "; la seconde fait référence au caractère évolutif de la démarche de recherche-intervention, permettant ainsi qu'une "marge d'initiative soit envisagée par la commande pour que des ajustements et des adaptations restent possibles " (ibid., p. 117). La formalisation de la commande est une étape clé de la recherche-intervention :

- elle lie contractuellement les acteurs engagés dans le projet ;

- elle explicite les attendus et les livrables ;

- elle fixe le montant des moyens financiers;

- elle pose le temps imparti. 
Ainsi, nous avions pour mission d'évaluer un dispositif de formation de façon concomitante à son développement, avec pour impératif de rendre tous les six mois un rapport confidentiel d'exécution de l'évaluation ainsi qu'une note d'étape destinée à être publiée sur le site : http://www.experimentation.jeunes.gouv.fr/. De plus, parmi les règles cadrant l'intervention, un comité de pilotage a été constitué par l'expérimentateur. Enfin, un rapport final d'évaluation devait clore l'intervention, proposant des pistes pour l'action (recommandations) et des conclusions quant à la généralisation du dispositif.

\section{D'une méthode mixte de recueil des données à la restitution des résultats}

Les deux derniers enjeux, méthodologique et praxéologique, vont être abordés ici. Ils sont dépendants des deux précédents ; cela signifie qu’ils ne pourront être atteints que si les enjeux ethique et stratégique ont été atteints au préalable.

\subsection{Produire des données mixtes suite à la négociation de la demande}

Cet enjeu méthodoligique est propre aux évaluations commanditées lorsque celles-ci préconisent une méthode de recueil et de traitement des données. Dans notre cas, l'approche valorisée était quantitative avec randomisation. Seulement, à la manière de Younès (op. cit.), nous nous inscrivons dans une approche écologique de l'évaluation de dispositif. Ainsi, la méthode recommandée ne donnait pas lieu à une connaissance complexe de l'objet étudié (prenant en compte les conditions environnementales de sa réalisation et le point de vue des acteurs impliqués). Ainsi, en amont, durant la phase de négociation, nous avons plaidé pour une méthode mixte de recueil des données, afin de répondre à la fois aux attendus de la demande et à nos orientations de recherche. Teddlie et Tashakkori (2003) rejettent la dichotomie quantitatif/qualitatif et préconisent une approche mixte ; celle-ci correspondant, selon eux, à une méthode pouvant apporter des résultats que des méthodes traditionnelles ne seraient pas en mesure de fournir. Creswell et Plano Clark (2011, p. 1) recensent différentes appellations de cette méthode : "troisième mouvement méthodologique ", " troisième paradigme de la recherche". Ils mentionnent que les premières définitions provenaient du champ de l'évaluation et ils définissent cette méthode comme comprenant «au moins une méthode quantitative (conçue pour recueillir des nombres) et une méthode qualitative (conçue pour recueillir des mots), où aucun type de méthode n'est intrinsèquement liée à un paradigme particulier» (ibid., p. 2).

Différentes associations des deux types de données existent. Une sera en particulier développée : "complementarity». Elle est définie comme "l'élaboration, la mise en valeur, l'illustration, et la clarification des résultats d'une méthode avec les résultats de l'autre méthode" (Greene, Caracelli et Graham in Creswell et Plano Clark, 2011, p. 62). Ainsi, dans notre 
recherche, la mixité est au cœur du modèle d'évaluation construit pour les dispositifs de formation.

\subsubsection{La triple articulation du modèle}

Afin de comprendre la pertinence de la méthode mixte, revenons aux fondements du modèle. Hadji (1989, p. 25) propose deux indicateurs, le "référent " qui correspond à "l'ensemble des normes ou critères qui serviront de grille de lecture de l'objet à évaluer", et le "référé" qui est associé à "ce qui sera retenu de cet objet à travers cette lecture " ${ }^{13}$.

Il présente une double articulation pour caractériser le processus d'évaluation. La première lie le référent et le référé afin de répondre à l'objectif de l'évaluation d'attribution d'une valeur. Il s'agit de mettre en perspective l'espéré et le réel. La seconde articulation concerne le référent et le référé avec "les réalités " (ibid., p. 26), soit une inscription dans le contexte.

Lecointe (1997) poursuit ce travail de conceptualisation et le complète. Il suggère de considérer les relations dynamiques d'une triple articulation entre référé, référent et référence. La référence permet, selon lui, de renoncer à l'équilibre mathématique faussé de la double articulation - la mesure d'un écart entre le désiré et le réel (l'attendu initial de la demande) - et introduit, à travers ce troisième indicateur, la valeur au sein de l'évaluation. Il faut entendre valeur dans le sens d'une attribution de sens à une action, intention, jugement, tous omniprésents dans l'évaluation. À partir des connaissances construites sur le dispositif (Agamben, 2007 ; Deleuze, 1988 ; Foucault, 1975), le modèle d'évaluation développé par Lecointe (1997) est appréhendé comme suit :

- le référent de l'évaluation coïncide avec les objectifs du dispositif ;

- le référé de l'évaluation s'apparente $\mathrm{au}(\mathrm{x})$ résultat(s) du dispositif, c'est-à-dire à la situation créée : l'obtenu, le réel observé en fonction du référent ;

- la référence de l'évaluation équivaut à la manière dont les chercheurs-évaluateurs se saisissent du dispositif pour attribuer un sens, une valeur au jugement évaluatif. À partir des connaissances établies ainsi qu'en fonction de notre positionnement épistémologique, la référence de l'évaluation accorde une importance aux vécus des acteurs.

Ces bases étant posées, nous pouvons à présent exposer plus finement le modèle d'évaluation.

13. Pour le formuler autrement, Hadji (ibid.), dans la distinction référent/référé, présente le référé comme les éléments d'une réalité et le référent comme l'idéal de cette réalité. 


\subsubsection{Les orientations du modèle d'évaluation de dispositif de formation}

Dans une approche en tension entre le modèle d'évaluation centré sur les produits et le modèle écologique, l'évaluation de dispositif de formation s'attache à mettre au jour les effets du dispositif au regard de phénomènes explicatifs, du point de vue des acteurs (de la préparation jusqu'à la mise en œuvre de l'expérimentation). Pour cela, l'évaluation s'est déroulée dans une approche concomitante. Ainsi, deux orientations ont été retenues : connaître pour objectiver les effets du dispositif, et connaître pour expliquer les effets du dispositif.

La première orientation, " objectiver ", est une demande souvent portée par les commanditaires ; elle permet de présenter un instantané d'une situation. L'objectif est de construire quantitativement des informations reflétant une situation observée. La seconde orientation, "expliquer ", vient compléter la première ; elle permet de l'interpréter par une connaissance fine du fonctionnement du dispositif de formation.

Ce modèle d'évaluation porte, d'une part, sur l'efficacité du dispositif car elle doit dire si les produits du dispositif sont bien en adéquation avec l'objectif. Cette idée d'efficacité ou de performance (Lafontaine, 2005) nous semble intéressante au regard de la perception des acteurs qui " habitent » le dispositif de formation. D'autre part, nous dissocions une mesure quantitative des effets (première orientation de l'évaluation) d'une description des effets produits par le dispositif sur les acteurs (seconde orientation de l'évaluation). Gardant à l'esprit la visée d'amélioration de la pratique, la seconde orientation du modèle permet d'expliquer les résultats de la mesure des effets. Elle permet de connaitre les phénomènes de mise en place (difficultés, réussite, stratégie d'adaptation) des acteurs. Ainsi, l'évaluation de dispositif de formation prend sens au regard des deux orientations qui se complètent dans la production des connaissances. Le protocole méthodologique a été conçu en intégrant, dès le départ, une approche quantitative et une approche qualitative, les deux approches ayant été déployées simultanément. 
Tableau 2. Synthèse de la méthode de recherche

\begin{tabular}{|c|c|c|c|c|c|}
\hline \multicolumn{2}{|c|}{ Hypothèses } & Variables & Indicateurs & $\begin{array}{l}\text { Techniques de } \\
\text { recueil }\end{array}$ & $\begin{array}{l}\text { Type de } \\
\text { données }\end{array}$ \\
\hline \multirow{3}{*}{$\begin{array}{l}\text { La participation } \\
\text { des étudiants au } \\
\text { dispositif contribue } \\
\text { à modifier leurs } \\
\text { représentations } \\
\text { (du marché de } \\
\text { l'emploi, du } \\
\text { fonctionnement de } \\
\text { l'entreprise, etc.) }\end{array}$} & $\begin{array}{l}\text { Il existe une relation } \\
\text { entre les actions des } \\
\text { animateurs et les } \\
\text { représentations des } \\
\text { étudiants }\end{array}$ & $\begin{array}{l}\text { Actions/ } \\
\text { Représentations }\end{array}$ & $\begin{array}{l}\text { Trajectoire d'orien- } \\
\text { tation, trajectoire } \\
\text { d'insertion, secteur } \\
\text { professionnel visé }\end{array}$ & $\begin{array}{c}\text { Questionnaires } \\
\text { (pré-test/post-test) } \\
\text { Comparaison groupe } \\
\text { expérimental (GE)/ } \\
\text { groupe témoin (GT) }\end{array}$ & Quantitatives \\
\hline & \multirow{2}{*}{$\begin{array}{l}\text { Il existe une relation } \\
\text { entre les éléments } \\
\text { du contexte et les } \\
\text { représentations des } \\
\text { étudiants }\end{array}$} & $\begin{array}{c}\text { Variables } \\
\text { individuelles } \\
\text { de l'étudiant/ } \\
\text { Représentations }\end{array}$ & $\begin{array}{l}\text { Sexe, âge, PCS } \\
\text { d'origine }\end{array}$ & $\begin{array}{c}\text { Questionnaire } \\
\text { (pré-test/post-test) } \\
\text { Comparaison GE/GT }\end{array}$ & Quantitatives \\
\hline & & $\begin{array}{l}\text { Variables } \\
\text { contextuelles de } \\
\text { l'étudiant/ } \\
\text { Représentations }\end{array}$ & $\begin{array}{l}\text { Environnement, la } \\
\text { formation, le lycée, } \\
\text { la classe de BTSA }\end{array}$ & $\begin{array}{c}\text { Questionnaire } \\
\text { (pré-test/post-test) } \\
\text { Comparaison GE/GT }\end{array}$ & Quantitatives \\
\hline \multirow{4}{*}{$\begin{array}{l}\text { L'activité } \\
\text { professionnelle } \\
\text { surtout dans un } \\
\text { contexte innovant, } \\
\text { contribue au } \\
\text { développement } \\
\text { professionnel (DP) } \\
\text { des animateurs }\end{array}$} & $\begin{array}{l}\text { II existe une relation } \\
\text { entre les actions des } \\
\text { animateurs et leur DP }\end{array}$ & Actions/DP & $\begin{array}{l}\text { Sentiment d'effica- } \\
\text { cité professionnelle } \\
\text { (SEP) }\end{array}$ & $\begin{array}{l}\text { Questionnaire ante } \\
\text { échelle } \\
\text { d'auto-efficacité }\end{array}$ & Quantitative \\
\hline & \multirow{3}{*}{$\begin{array}{l}\text { Il existe une relation } \\
\text { entre les éléments du } \\
\text { contexte et le DP des } \\
\text { animateurs }\end{array}$} & $\begin{array}{l}\text { Variables indi- } \\
\text { viduelles des } \\
\text { animateurs/DP }\end{array}$ & $\begin{array}{l}\text { Sexe, âge, statut, } \\
\text { diplôme(s), } \\
\text { expérience }\end{array}$ & Questionnaire ante & \multirow{3}{*}{ Qualitative } \\
\hline & & $\begin{array}{c}\text { Variables } \\
\text { contextuelles des } \\
\text { animateurs/DP }\end{array}$ & $\begin{array}{l}\text { Environnement, } \\
\text { formation, lycée, } \\
\text { classe de BTSA }\end{array}$ & Questionnaire & \\
\hline & & $\begin{array}{l}\text { Pratiques de mise } \\
\text { en œuvre du } \\
\text { dispositif/ DP }\end{array}$ & $\begin{array}{c}\text { Analyse de } \\
\text { l'activité par les } \\
\text { animateurs }\end{array}$ & $\begin{array}{l}\text { Observations } \\
\text { Entretiens d'auto } \\
\text { confrontations } \\
\text { (années } 1 \text { et 2) }\end{array}$ & \\
\hline $\begin{array}{l}\text { L'efficacité perçue } \\
\text { des étudiants du } \\
\text { dispositif en lien } \\
\text { avec le SEP des } \\
\text { animateurs }\end{array}$ & $\begin{array}{l}\text { Les animateurs ayant } \\
\text { un SEP élevé seront } \\
\text { ceux pour lesquels les } \\
\text { étudiants exprimeront } \\
\text { une plus forte effica- } \\
\text { cité percue }\end{array}$ & \multicolumn{2}{|c|}{$\begin{array}{l}\text { Efficacité des animateurs perçue par les } \\
\text { étudiants du GE/SEP }\end{array}$} & $\begin{array}{l}\text { Observations } \\
\text { Entretiens semi- } \\
\text { directifs question- } \\
\text { naires SEP }\end{array}$ & $\begin{array}{l}\text { Qualitative } \\
\text { Quantitative }\end{array}$ \\
\hline
\end{tabular}

PCS : profession et catégorie sociale. BTSA : Brevet de technicien supérieur agricole.

Source : Auteure.

\subsection{Accompagner la décision politique par la restitution des résultats}

A présent, la question du statut des résultats produits dans une recherche commanditée, de type accompagnement à la décision politique, va être abordée. La production d'une recherche-intervention est certes destinée à la communauté scientifique mais surtout aux communautés des décideurs et des praticiens-expérimentateurs.

Apportons d'abord quelques éléments sur l'aide à la décision politique (ADP), théorie à l'aune de laquelle nous développons l'accompagnement à la décision politique. L'ADP est une réponse apportée à une demande médiatisée par une institution décisionnaire. 
Elle se définit comme "une double activité d'expertise et de conseil "14 (Bedin, 1999, p. 90). Ainsi, cette "pratique d'intervention à destination des sujets-décideurs" (Bedin, 1993, p. II) comprend un processus d'aide pris en charge par une personne extérieure à l'organisation porteuse de la demande. Le passage de l'aide à la décision politique à l'accompagnement de la décision politique réside dans une posture du chercheurintervenant qui consiste à traduire son action et ses résultats aux décideurs, mais aussi aux partenaires.

Si cette pratique n'est pas nouvelle, soulignons deux points primordiaux qui concernent la traduction de l'action et des résultats. Le premier consiste à assurer la diffusion des résultats en dehors des communautés décisionnaires et politiques. Le second, extension du premier, concerne la restitution des savoirs produits auprès des partenaires (ici praticiens-expérimentateurs).

En effet, lors de la restitution, les chercheurs-intervenants "remettent " leurs résultats et en sont ainsi dépossédés; ils adressent des savoirs, sous la forme de rapports, dont la destinée est inconnue. Ainsi, l'accompagnement de la décision politique ne se définit pas uniquement comme une pratique à destination des décideurs.

Tableau 3. Synthèses des productions et de leur restitution au cours de la rechercheintervention (2009-2013)

\begin{tabular}{|c|c|c|}
\hline Type de productions & Destinataires & Objet de la restitution \\
\hline Rapports intermédiaires & Décideurs (FEJ) & \multirow{3}{*}{$\begin{array}{l}\text { Avancement du projet et progression des } \\
\text { résultats }\end{array}$} \\
\hline \multirow{3}{*}{ Exposés oraux } & Exposé intermédiaire : ensemble des partenaires praticiens & \\
\hline & $\begin{array}{l}\text { Exposé intermédiaire : comité de pilotage de l'expérimentation } \\
\text { sociale (expérimentation et évaluation) }\end{array}$ & \\
\hline & $\begin{array}{l}\text { Exposé final : ensemble des partenaires } \\
\text { praticiens-expérimentateurs }\end{array}$ & Résultats finaux et recommandations \\
\hline Entretien sociologique & $\begin{array}{l}\text { Partenaires praticiens-expérimentateurs ayant été sélec- } \\
\text { tionnés et ayant accepté de participer au recueil de données } \\
\text { (observations et entretiens d'auto-confrontation) }\end{array}$ & $\begin{array}{l}\text { Validation des conclusions de la recherche } \\
\text { concernant leur développement } \\
\text { professionnel }\end{array}$ \\
\hline Rapport final & $\begin{array}{l}\text { Décideurs (FEJ), partenaires praticiens et une partie de la } \\
\text { communauté scientifique (jury de thèse), le rapport figurant } \\
\text { dans les annexes de la thèse et une note de synthèse de ce } \\
\text { rapport ayant été intégré à la thèse. }\end{array}$ & Résultats finaux et recommandations \\
\hline
\end{tabular}

FEJ : Fonds d'expérimentations pour la jeunesse.

Source : Auteure.

14. Cette double activité "se compose de différentes séquences : l'analyse de la demande portée par un sujet politique; l'élaboration d'un pré-projet ; la formalisation des termes du contrat d'intervention (commande); la réalisation d'enquêtes de terrain par contact direct; la restitution d'un rapport d'expertise-conseil à la (aux) structure(s)-commanditaire(s), voire aux différents groupes sociaux concernés par le projet ", Bedin (ibid.). 
La nécessité d'accompagnement est en lien avec deux enjeux que nous avons abordés supra. En effet, cela renvoie à l'enjeu éthique car les partenaires (expérimentateurs), parties prenantes de ce travail, ne sauraient être exclus de ces conclusions. Ensuite, cela renvoie à l'enjeu stratégique et à l'importance d'impliquer davantage les expérimentateurs afin d'éviter que les résultats ne soient réinterprétés en fonction, par exemple, d'enjeu électoraux, s'ils n'étaient destinés qu'aux décideurs.

\section{Conclusion}

Cette contribution a présenté une démarche spécifique de recherche, lorsque celle-ci se trouve en situation de commande en partenariat avec une instance politique. Nous avons étudié le cas d'une recherche-intervention dont l'objectif était l'évaluation d'un dispositif de formation.

L'évaluation ici procède d'une logique en tension entre reddition des comptes et développement des acteurs et du dispositif.

À partir d'un questionnement sur la posture et l'autonomie d'une équipe de recherche dans cette situation de travail, nous avons développé quatre enjeux : éthique, stratégique, méthodologique et praxéologique, en lien avec la recherche que nous avons menée.

Aussi, les réflexions développées ne pourraient être généralisables et se veulent un témoignage et un point de discussion (et de controverse) au sein de la communauté scientifique. Ces enjeux sont interdépendants et s'inscrivent dans une logique processuelle. En effet, l'enjeu éthique correspond à l'engagement et à la posture du chercheur dans ce type de projet ; il implique ensuite l'enjeu stratégique grâce auquel une phase de négociation des termes de la recherche peut être menée. Ensuite, l'enjeu méthodologique, porté par le précédent, permet de mettre en place la méthode de recherche négociée avec les commanditaires et renforce l'autonomie de la posture des chercheurs-intervenants. Enfin, l'enjeu praxéologique porte la question du statut des résultats et de leur restitution. Cette question nous paraît essentielle pour accompagner la décision, qu'elle soit politique ou d'une autre nature. Nous aimerions insister sur les différents niveaux de la restitution, c'est-à-dire l'importance de l'adresser aux différents types d'acteurs, qu'ils soient décisionnaires ou simplement partenaires de la recherche-intervention. 


\section{Bibliographie}

Agamben G. (2007), Qu'est-ce qu’un dispositif?, Paris, Payot \& Rivages.

Ardoino J. (1990), Les postures (ou impostures) respectives du chercheur, de l'expert et $d u$ consultant. Les nouvelles formes de la Recherche en éducation au regard d'une Europe en devenir, AFIRSE (Association francophone internationale de recherche scientifique en éducation), Actes du colloque, "Les nouvelles formes de la recherche en éducation au regard d'une Europe en devenir ", Alençon, 24-26 mai.

Audran J. (2007), "Dispositifs et situations, quelles articulations ?", Questions vives, 4(8), pp. 12-15.

Bedin V. (1993), L'aide à la décision politique, de la recherche à la praxéologie : le cas de l'expertise-conseil, thèse de doctorat en sciences de l'éducation, Toulouse.

Bedin V. (2007), "Faire le choix du concept d' "évaluation-conseil” en éducation et formation", Les dossiers des sciences de l'éducation, (18).

Bedin V. \& Talbot L. (2010), « Réinterroger la commande d'évaluation des dispositifs d'éducation ou de formation : nouvelles propositions, nouveaux enjeux ", Présenté à Actualité de la recherche en éducation et en formation, université de Genève.

Broussal D. (2010), "Evaluation des dispositifs de formation des professions paramédicales dans un contexte d'universitarisation : quelle place pour la professionnalité ? ", Présenté à Actualité de la recherche en éducation et en formation, université de Genève.

Bru M. (1998), Qu'y a-t-il à prouver quand il s'agit d'éducation ?, in Hadji C. \& Baillé J. (Éd.), Recherche et éducation. Vers une nouvelle "alliance". La démarche de preuve en 10 questions, Paris, Bruxelles, De Boeck Université, pp. 45-65.

Castel R. (2004), "La sociologie et la réponse à la demande sociale ", in A quoi sert la sociologie?, Paris, La Découverte, pp. 67-77.

Creswell J. W. \& Plano Clark V. L. (2011), Designing and conducting mixed methods research, Los Angeles, Sage.

De Ketele J.-M. de. (1993), «L'évaluation conjuguée en paradigmes ", Revue française de pédagogie, 103(1), pp. 59-80.

Deleuze G. (1988), Qu'est-ce qu'un dispositif? in Michel Foucault. Rencontre internationale, Paris 9, 10-11 janvier, Paris, Le Seuil, pp. 316-325.

Desgagné S. (1997), "Le concept de recherche collaborative : l'idée d'un rapprochement entre chercheurs universitaires et praticiens enseignants ", Revue des sciences de l'éducation, 23(2), pp. 371-393. 
Duru-Bellat M. \& Jarousse J.-P. (2001), " Portée et limites d'une évaluation des politiques et des pratiques éducatives par les résultats ", Education et Sociétés, 2(8), pp. 97-109.

Dussault M. (2008), "Evaluation des programmes et des dispositifs éducatifs ", Mesure et évaluation en éducation, 30(3), pp. 61-62.

Figari G. (2008), «L'évaluation des dispositifs éducatifs ", Mesure et évaluation en éducation, 31(3), pp. 77-93.

Foucault M. (1975), Surveiller et punir, Paris, Gallimard.

Hadji C. \& Baillé J. (Éd.). (1998), Recherche et éducation : vers "une nouvelle alliance", la démarche de preuve en 10 questions, Bruxelles Paris, De Boeck université.

Lafontaine D. (2005), "Relativiser les performances des établissements scolaires : quelle valeur ajoutée ? ", in Demeuse M., Baye A., Straeten M.-H., Nicaise J. \& Matoul A. (Éd.), Vers une école juste et efficace, Bruxelles, De Boeck, pp. 519-538.

Lafontaine D. \& Simon M. (2008), "Evaluation des systèmes éducatifs ", Mesure et évaluation en éducation, 31(3), pp. 95-123.

Lecointe M. (1997), Les enjeux de l'évaluation, Paris Montréal, L'Harmattan.

Marcel J.-F. (2010), “Des tensions entre le "sur” et le "pour" dans la recherche en éducation : questions(s) de posture(s) ", Cahiers du CERFEE, (27-28), pp. 41-64.

Marcel J.-F. \& Nunez Moscoso J. (2012), «La figura del investigador ciudadano : hacia un (re)encuentro con el ethos de la investigacion en education ", Revista estudios cooperativos, $17(1$ y 2$)$, pp. 101-121.

Mohd Suki N. (2011), "Using mobile device for learning : from student's perspective ", US-China Education Review, (1), pp. 44-53.

Mons N. (2008), "Evaluation des politiques éducatives et comparaisons internationales ", Revue française de pédagogie, (164), pp. 5-13.

Peraya D. \& Peltier C. (2012), «Une année d'immersion dans un dispositif de formation aux technologies : prise de conscience du potentiel éducatif des TICE, intentions d'action et changement de pratique ", Revue internationale des technologies en pédagogie universitaire, 9(1-2), pp. 111-135.

Perret B. (2001), L'évaluation des politiques publiques, Paris, La Découverte.

Pesche D. (2012), Faire de la recherche et pratiquer l'expertise dans une logique d'accompagnement, in "Les relations entre experts, chercheurs et acteurs associatifs face au développement des organisations collectives d'agriculteurs dans les filières agricoles ", Toulouse, MSHS-T/INRA, pp. 9-17.

Ricœur P. (1996), Soi-même comme un autre, Paris, Éd. du Seuil. 
Saussez F. \& Lessard C. (2009), «Entre orthodoxie et pluralisme, les enjeux de l'éducation basée sur la preuve ", Revue française de pédagogie, (168), pp. 111-136.

Teddlie C. \& Tashakkori A. (2003), " Major issues and controversies in the use of mixed methods in the social and behavorial sciences ", in Handbook of mixed methods in social and behavioral research Thousand Oaks, Sage Publications, pp. 3-50.

Wong-Ratcliff M., Powell Ezell S., Cage B. N. \& Chen C. C. (2011), "The reading first program and statewide-mandated assessments : a three-year comparative study ", Journal of Modern Education Review, 1(1), pp. 22-33.

Younès N. (2010), "L'évaluation " écologique " des dispositifs éducatifs et de formations comme perspective ", Présenté à Actes du congrès de l'Actualité de la recherche en éducation et en formation, université de Genève. 


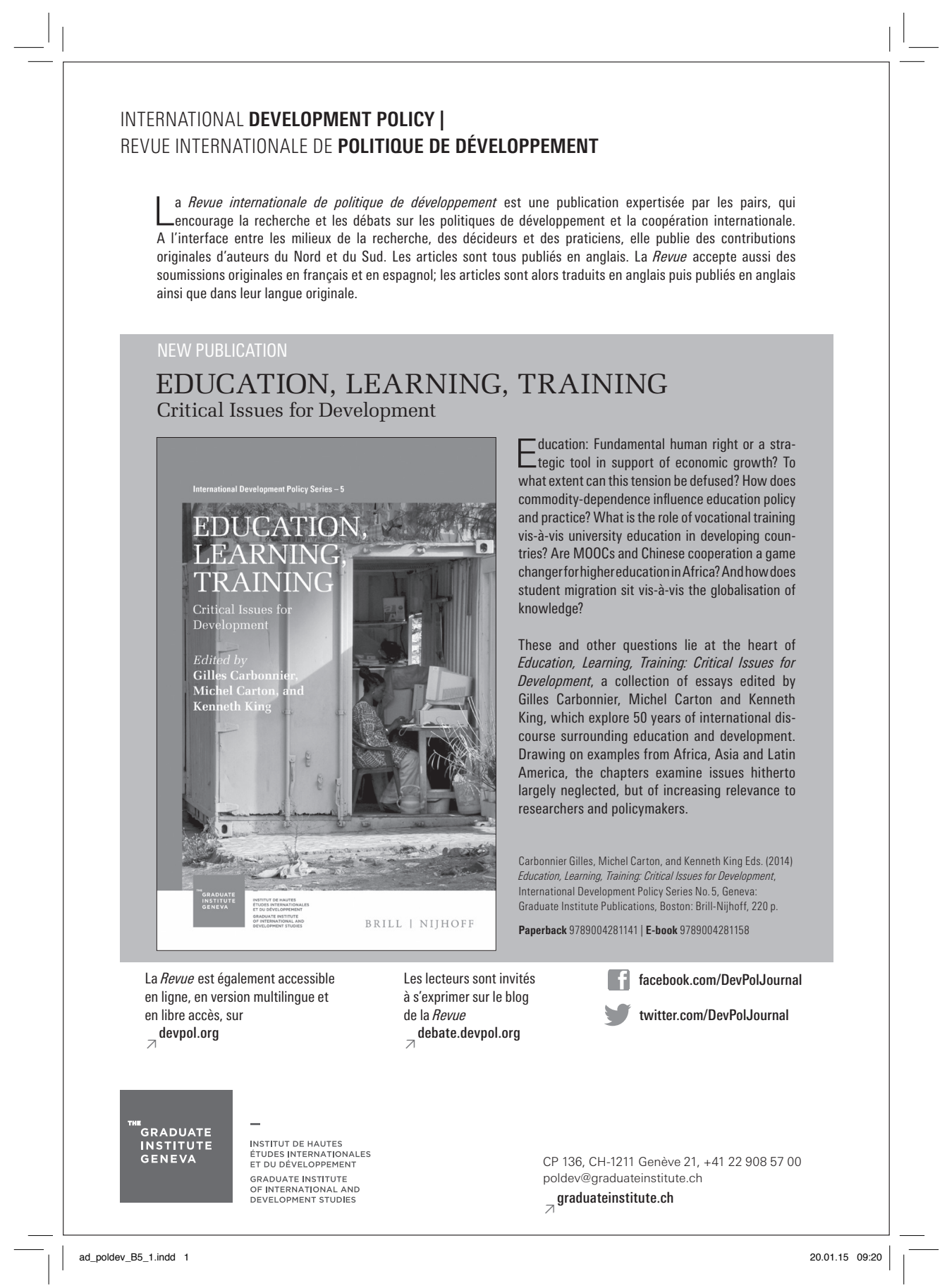

